\title{
One-hour loop-mediated isothermal amplification assay for the detection of quarantinable toxigenic Fusarium garmanirum
}

\author{
Hassan Almoammar ${ }^{1,2^{\star}}$, Ali H. Bahkali ${ }^{2,3}$ and Kamel A. Abd-Elsalam ${ }^{4}$ \\ ${ }^{1}$ King Abdulaziz City for Science and Technology (KACST), P. O. Box 6086, Riyadh 11442, Kingdom of Saudi Arabia. \\ ${ }^{2}$ Botany and Microbiology Department, Faculty of Science, King Saud University, Riyadh, Saudi Arabia. \\ ${ }^{3}$ Center of Excellence in Biotechnology Research, King Saud University, P.O. Box 2455, Riyadh 11451, Saudi Arabia. \\ ${ }^{4}$ Unit of Excellence in Nano-Molecular Plant Pathology Research (ARC), Egypt.
}

Accepted 25 March, 2013

\begin{abstract}
Fusarium head blight (FHB) caused by several Fusarium species is one of the most serious diseases affecting wheat throughout the world. The in vitro production of the toxins deoxynivalenol, zearalenone fumonisin, T-2, and HT-2 was quantitvely evaluated in 8 different isolates of Fusarium species collected from feed samples. It was possible to detect zearalenone and the other mycotoxins in $100 \%$ and $50 \%$ of the isolates, respectively. In the present study, loop-mediated isothermal amplification method (LAMP) was designed for diagnosing Fusarium garmanirum infections and testing against feed samples, infested samples and pure cultures. The LAMP amplicon was directly visualized in the reaction tubes by the naked eye following the addition of calcein fluorescence. The LAMP products appeared as DNA marker pattern, with many bands of different sizes from 145 base pairs up to the loading well. Loop-LAMP procedure was used to detect genomic DNA of $F$. graminearum in fungal pure culture and in contaminated feed samples. In the future, this assay will support plant quarantine programs in Saudi Arabia and Gulf Cooperation Council states, to prevent the introduction of foreign FHB species.
\end{abstract}

Key words: LAMP-PCR, Fusarium head blight, feed samples.

\section{INTRODUCTION}

Head blight is caused by several Fusarium spp.; distribution and predominance of species significantly vary among climatic conditions, geographical zones, countries, and years (Doohan et al., 2003; Xu, 2003). Morphological identification of Fusarium spp. is difficult due to their similarities. For example, F. avenaceum are very difficult to separate by the morphological characteristics of their spores (Yli Mattila et al., 2004). Recently, the novel nucleic acid amplification method loop-mediated isothermal amplification (LAMP) has been reported as a simple rapid diagnostic tool for early detection of microorganisms (Parida et al., 2008). LAMP was also developed for rapid detection of pathogenic or allergenic fungal in the environment (Sun et al., 2010). Recently, LAMP-PCR using internal labeled probes have been developed for the major FHB pathogens in different plant materials (Niessen and Vogel, 2010; Abd-Elsalam et al., 2011; Niessen et al., 2012). LAMP depends on using a set of 
Table 1. Production of mycotoxin by Fusarium spp. isolated from feed samples.

\begin{tabular}{lccccc}
\hline \multirow{2}{*}{ Isolate } & \multicolumn{5}{c}{ Mycotoxins $(\boldsymbol{\mu g} / \mathbf{k g})$} \\
\cline { 2 - 6 } & Fumonisin & Zearalenone & T-2 & DON & HT-2 \\
\hline F. semitectum & 0 & 85 & 0 & 0 & 0 \\
F. semitectum & 0 & 50 & 0 & 0 & 0 \\
F. semitectum & 0 & 60 & 0 & 0 & 0 \\
F. semitectum & 0 & 130 & 0 & 0 & 0 \\
F. verticillioides & 1050 & 245 & 8.0 & 640 & 3.0 \\
F. graminearum & 850 & 354 & 2.0 & 550 & 0 \\
F. graminearum & 700 & 420 & 4.0 & 320 & 0 \\
F. graminearum & 450 & 557 & 2.0 & 120 & 0 \\
\hline
\end{tabular}

six oligonucleotide primers with eight binding sites hybridizing specifically to different regions of a target gene, and a thermophilic DNA polymerase from $F$. garmanirum for DNA amplification (Niessen and Vogel, 2010; Abd-Elsalam et al., 2011). This methods has been used to detect plant pathogenic fungi like Phytophthora ramorum (Tomlinson et al., 2007), Phytophthora sojae (Dai et al., 2012), A. flavus and A. parasiticus (Ahmed et al., 2010), toxigenic Fusarium (Denschlag et al., 2012; Niessen et al., 2012). The current study describes the LAMP for the detection of $F$. garmanirum in infested feed sample.

\section{MATERIALS AND METHODS}

\section{Feed Samples}

Twenty samples of fodder used in camel feeding in Saudi Arabia were collected. Samples including natural feed (14 samples) and compound feed (6 samples), were collected from various animal feed factories, storehouse, and fodder markets from vendor or distributors during 2009.

\section{Isolation and identification of Fusarium spp.}

Feed sample were surface-sterilized for 1 min with a $1 \%$ sodium hypochlorite solution, rinsed twice in sterile distilled water and dried in a laminar flow cabinet. One growth media, Potato Dextrose Agar (PDA) was used for fungal isolations. The plates were incubated at $28^{\circ} \mathrm{C}$ in the dark for 5-7 days. All Fusarium isolates were subcultured on PDA, Spezieller Nahrstoffarmer Agar (SNA) using a single spore technique (Leslie and Summerell, 2006). The fungal cultures were maintained at $10^{\circ} \mathrm{C}$ on SNA medium $\left(0.5 \mathrm{~g} \mathrm{I}^{-1} \mathrm{MgSO}_{4} \times 7\right.$ $\mathrm{H} 2 \mathrm{O}, 1 \mathrm{~g} \mathrm{I}^{-1} \mathrm{KNO}_{3}$ (Roth, Karlsruhe, Germany), $0.2 \mathrm{~g} \mathrm{I}^{-1}$ sucrose, 0.2 $\mathrm{g} \mathrm{l}-1$ glucose, $0.5 \mathrm{~g} \mathrm{l}^{-1} \mathrm{KCl}, 1 \mathrm{gl}^{-1} \mathrm{KH} 2 \mathrm{PO} 4$ and $15 \mathrm{~g} \mathrm{l}^{-1}$ agar (Merck, Darmstadt, Germany). Fungal and Fusarium isolates used in the current study are listed in Table 1.

\section{Feed samples infestation}

Plant Pathology Research Institute (PPathRI) strain of F. graminearum were inoculated on PDA slants and incubated for 2-4 weeks and spores were harvested as previously reported by Hilton et al. (1999). Feed samples were infested by placing $100 \mu \mathrm{L}$ of $F$. graminearum conidial suspension (containing 106 conidia) on each gram feed sample. Control sample were treated with $100 \mu \mathrm{L}$ of sterile distilled water. Inoculated sample were incubated in a moist chamber for $72 \mathrm{~h}$ and returned to the growth chamber at $21^{\circ} \mathrm{C}$ (day) $/ 16^{\circ} \mathrm{C}$ (night) temperatures with day/night regime of $16 \mathrm{~h} / 8 \mathrm{~h}$.

\section{Fusarium mycotoxins}

Fusarium mycotoxins (Fumonisin, HT-2, Zearalenone, T-2, Neosolaniol and DON) content was determined using the VICAM (1998) method. The method was similar with all former toxins except the dilution buffer, developer and immunoaffinity column. Each isolate was grown in elmnyer flask $100 \mathrm{ml}$ on SMKY media. The incubation period was 7 days at $25 \pm 2^{\circ} \mathrm{C}$. After blending on high speed for $1 \mathrm{~min}$ with $5 \mathrm{~g}$ of sodium chloride. Twenty milliliter of culture filtrate was added to $80 \mathrm{ml}$ of methanol (HPLC grad) and filtered through a fluted filter paper. The extract $(10 \mathrm{ml})$ was diluted with $40 \mathrm{ml}$ of phosphate buffered saline (PBS) $/ 0.1 \%$ Tween-20 wash buffer and filtered through a 1.0-Am microfiber filter. The diluted extract was passed through the column, which was washed with $10 \mathrm{ml}$ of PBS $/ 0.1 \%$ Tween-20 wash buffer followed by $10 \mathrm{ml}$ of PBS. Fumonisins were eluted from the column with $1 \mathrm{ml}$ HPLC grade methanol. A mixture of developer A and developer B $(1 \mathrm{ml})$ was added to the elute collected in a cuvette that was placed in a fluorometer (VICAM Fluorometer Series 4, Watertown, USA) for fumonisin measurement.

\section{Fungal mats}

The Fusarium spp. isolates and fungal cultures obtained from Saudi MycoBank for DNA isolation were grown for 8 days in $100 \mathrm{ml}$ Potato Dextrose Broth (PDB, $24 \mathrm{~g} \mathrm{I}^{-1}$; Scharlau, Barcelona, Spain). Fungal mycelium mats $(100 \mathrm{mg})$ from 3 -day-old colonies grown on duplex agar medium was homogenized by an electric grinder prior to DNA extraction. One hundred milligram mycelium was scraped off the agar using a spatula.

\section{DNA extraction}

A hand-operated or electric grinder (Retsch, Germany) was used to homogenize fresh mycelium in a $1.5-\mathrm{mL}$ microfuge tube. To prevent cross-contamination, the pestle tip was changed and sterilized between isolations (the pestle tip was immersed in $70 \% \mathrm{EtOH}$ and the grinder was turned on for $3 \mathrm{~s}$ and subsequently the pestle tip was rinsed in sterile distilled water and dried with sterilized filter paper. Pre-warmed (at $\left.65^{\circ} \mathrm{C}\right) 600 \mu \mathrm{L}$ of DNA extraction buffer $(200$ $\mathrm{mM}$ Tris- $\mathrm{HCl}, \mathrm{pH} 8.5,250 \mathrm{mM} \mathrm{NaCl}, 25 \mathrm{mM}$ EDTA, 0.5\% SDS, $1 \%$ PVP) was added to homogenized samples. Six microliter of RNase A $(20 \mu \mathrm{g} / \mathrm{ml})$ was added and microtubes were incubated at $65^{\circ} \mathrm{C}$ in hot water bath for 15 min with irregular shaking and swirling for every $5 \mathrm{~min}$. For protein precipitation $160 \mu \mathrm{l}$ was added (3 M so- 
Table 2. LAMP primers list used in the current study.

\begin{tabular}{llc}
\hline Primer code & Primer Sequence & Concentration \\
\hline FIP-gaoA ID4 & $\begin{array}{l}\text { 5'-CGC AAG TGA CGG CCC AGT TGC } \\
\text { TTC GAG CCT CAG CAC CTA-3' }\end{array}$ & $1.6 \mathrm{mM}$ \\
BIP-gaoA ID4 & 5'-TGC AAC AAG GCC ATT GAT GGC CGT & $1.6 \mathrm{mM}$ \\
& TGG CGC CAT AGA ATG T-3' & \\
F3-gaoA ID4 & 5'-AGG GAG TCT TCA GTT CCT GA-3' & $0.2 \mathrm{~m} \mathrm{M}$ \\
B3-gaoA ID4 & 5'-GTG AGG GGG CTT TGG ATC-3' & $0.2 \mathrm{~m} \mathrm{M}$ \\
LoopF-gaoA ID4 & 5'-GTT GCG AGA AAT GGC GCT TCC G-3' & $0.8 \mathrm{mM}$ \\
LoopB-gaoA ID4 & 5'-ACA AGG ATA CCT TTT GGC AC-3' & $0.8 \mathrm{mM}$ \\
\hline
\end{tabular}

Niessen and Vogel (2010). All nucleotides were purchased from Eurofins MWG Operon, Ebersberg, Germany.

dium acetate, $\mathrm{pH}$ 5.3) and mixed thoroughly by inverting the tube. The mixture was centrifuged at $10,000 \mathrm{rpm}$ for $8 \mathrm{~min}$ at room temperature. The supernatant was carefully decanted and transferred to a fresh tube and was precipitated with equal volumes of cold Isopropanol (molecular biolgy grade), and quietly mixed to generate fibrous DNA and incubated at room temperature for $10 \mathrm{~min}$. Genomic DNA was collected by centrifuging at $10000 \mathrm{rpm}$ for $10 \mathrm{~min}$. The resulting pellet was washed twice with $80 \%$ ethanol and then was centrifuged at $8000 \mathrm{rpm}$ for $2 \mathrm{~min}$. The pellet so obtained has been dried under vacuum using Vacufuge Concentrators 5301 (Eppendorf) at $37^{\circ} \mathrm{C}$ for $5 \mathrm{~min}$. In the final step, DNA was eluted in $100 \mu \mathrm{L}$ of sterile TE buffer (10 mM Tris-HCl, $1 \mathrm{mM}$ EDTA pH 8.0) and kept at $-20^{\circ} \mathrm{C}$ until used as template for PCR amplification (Abd-Elsalam et al. , 2011).

\section{LAMP reaction}

We used six primers designed by Niessen and Vogel (2010) with nucleic acid sequences and molarities concentration as shown in Table 2. The LAMP assay was carried out in a total of $25 \mu \mathrm{l}$ reaction mixture containing 10× Bst-DNA polymerase buffer (2.5 mM each), betaine $(10 \mu \mathrm{M}$ each), deoxynucleotide triphosphates $(2.5 \mathrm{mM}$ each), $\mathrm{MgSO}_{4}$ (10 mM each), FIP and BIP (1.6 mM each), F3 and B3 primers (0.2 mM each), loop-F and loop-B ( $0.8 \mathrm{mM}$ each) (Table 2), Bst DNA polymerase (8 $\mathrm{U}$ ) (New England BioLabs), dd $\mathrm{H}_{2} \mathrm{O}$, and template DNA $(1 \mu \mathrm{l})$. Before incubation $25 \mu \mathrm{M}$ calcein and $0.5 \mathrm{mM}$ $\mathrm{MnCl}_{2}$ were added to each $25 \mu \mathrm{l}$ reaction mix. The template DNA was omitted in one reaction for negative control. LAMP reactions were carried out at $63^{\circ} \mathrm{C}$ for 60 min using Swift Spectrum 48 Thermal Cycler (ESCO, Singapore). Visual inspection of the LAMP amplicons in the reaction tube were performed by adding fluorescent detection reagent before the incubation of the reaction tubes, the fluorescent signals of the solutions were observed under UV light. Direct visualization of amplified product was achieved by separation of the LAMP reactions on $1.5 \%$ agarose gels following incubation. Gels were run at $60 \mathrm{~V}, 55 \mathrm{~mA}$ for $45 \mathrm{~min}$ and stained with ethidium bromide solution (1 $\mathrm{g} / \mathrm{mL})$. PCR amplicons were documented using a Biorad Gel Doc System (Biorad, NSW, Australia).

\section{RESULTS}

\section{Screening of Fusarium spp. for mycotoxin production}

Toxin-producing isolates varied in the type and concentrations of toxin produced. F. verticillioides was the highest producer of fumonisin, zearalenone and DON (1050,
245 , and $640 \mu \mathrm{g} / \mathrm{kg}$, respectively). F. semitectum only produces zearalenone toxin (Table 1). $F$. graminearum isolates were not able to produce HT-2. Fumonisins were produced by a number of Fusarium species, notably $F$. verticillioides and $F$. graminearum.

\section{LAMP for detecting $F$. graminearum contamination in feeds}

The LAMP-PCR technique uses four primers that identified six regions on the target nucleic acid, so that the specificity is particularly high. For this reason, in the LAMP method, detection can be done without inspection of the amplification product during or after the amplification. Visualization of DNA amplification during LAMP was achieved by inspection of the reaction vessels under UV $366 \mathrm{~nm}$ trans-illumination after adding manganese quenched calcein to the master mix. Intensity of the fluorescence signal was independent from DNA concentrations in positive samples. Samples with water added instead of DNA showed no fluorescence under these conditions. The size of the LAMP reaction products ranged from 145 bp up to the loading well on the agarose gel, indicating specific amplification of the target sequence. Positive results were achieved with $F$. graminearum isolates, while all reactions with Fusarium and fungal species included for comparison remained negative, with a response identical to the negative control. Among the strains of Fusarium spp., a fluorescent signal was obtained from four tested $F$. graminearum isolates. Testing of other Fusarium spp. and species from other genera revealed no fluorescent signal in the LAMP assay. No cross reactivity was observed with other fungal DNA $F$. semitectum (lane 11), 12= F. verticillioides (lane 12), Aspergillius (lane 13), Pencillium (lane 14) (Figures 1 and 2).

\section{DISCUSSION}

Conventional diagnostic methods for the detection and identification of $F$. graminearum in culture or in infected grains are based on micro and macro morphological 


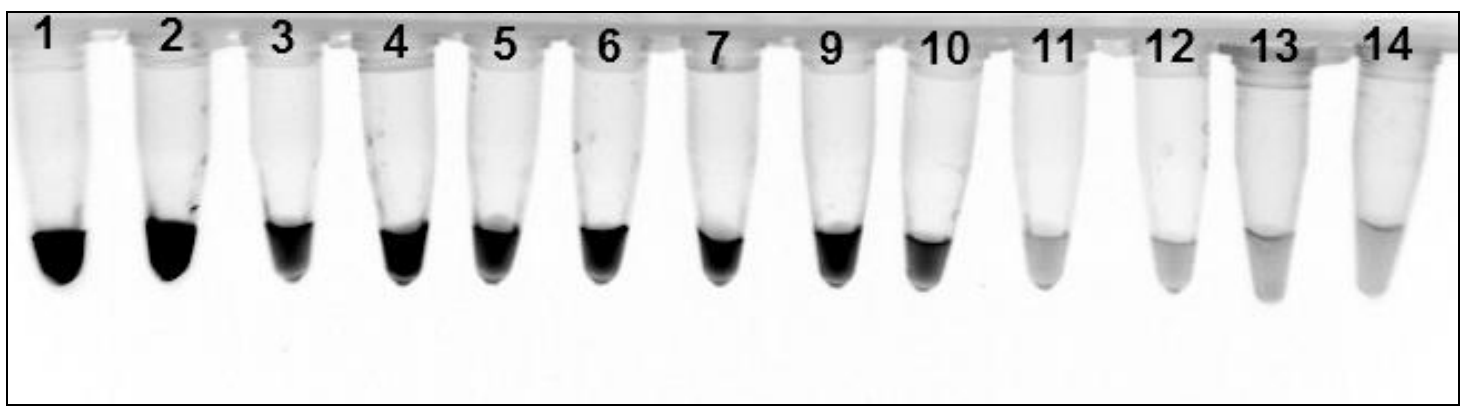

Figure 1.Visual appearance of LAMP-PCR products by using primer set gaoA ID4 and Calcein fluorescence under UV $366 \mathrm{~nm}$ light. The reactions were incubated in a water bath for $60 \mathrm{~min}$ at $63^{\circ} \mathrm{C} .1=F$. graminearum, $2=F$. graminearum, $3=F$. graminearum, Infested feed samples with $F$. graminearum (PPathRI 0555) were included; 4=Wheat bran, 5=Wheat bran, 6=Barley grain, $7=$ fresh barely, $8=$ Crushed corn, $9=$ Sorghum, $10=$ Millet Grain. 11= F. semitectum, 12=F. verticillioides, 13=Aspergillius, $14=$ Pencillium. PPathRI $=$ Plant Pathology Research Institute.

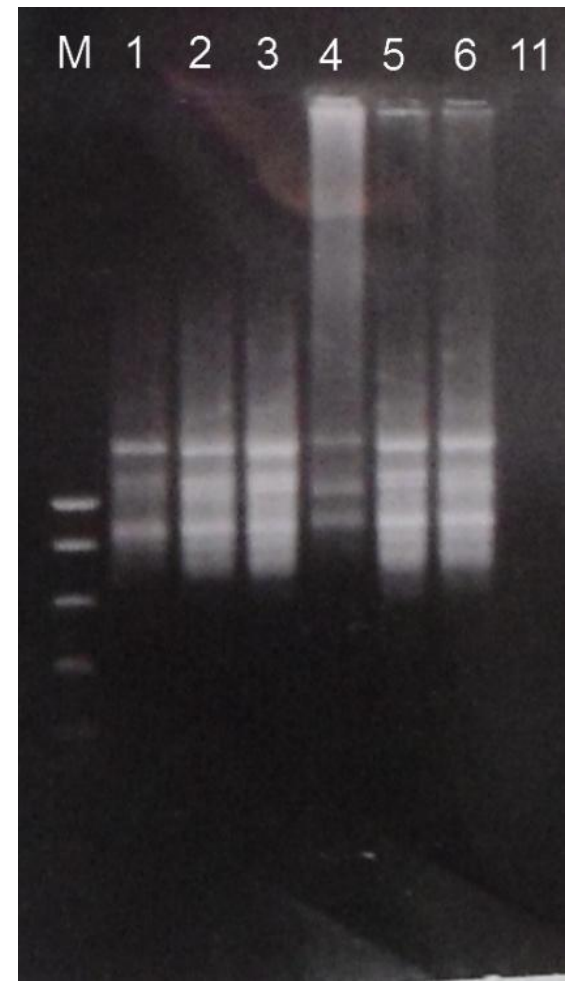

Figure 2. Agarose gel electrophoresis of loop-mediated isothermal amplification products amplified from genomic DNA extacted during the current study. $1=F$. graminearum, 2=F. graminearum, $3=F$. graminearum, Infested feed samples with F. graminearum (PPathRI 0555) were included; $4=$ Wheat bran, $5=$ Wheat bran, $6=$ Barley grain, $11=F$. semitectum.

features. These methods are time consuming, require experience, and are difficult to distinguish between similar species.
Toxin-producing isolates varied in the type and concentrations of toxin produced. $F$. verticillioides was the highest producer of fumonisin, Zearalenone and DON. The very high levels of $F$. verticillioides and $F$. semitectum in feed samples are known to infect crops in the field and propagate at moisture contents. This fungus is known for the production of fumonisins, of which fumonisin $B 1$ is the most important.

LAMP-PCR using the gaoA gene was used to detect $F$. graminearum in contaminated feed products. The method developed by Niessen and Vogel (2010) is cost-effective, high throughput quantification of $F$. graminearum in wheat grains. The LAMP assay is virtually equipment-free, requiring only a simple device such as a water bath to maintain a constant isothermal temperature. It is therefore much less technically demanding than nested PCR. Detectable copies of DNA were obtained after only $1 \mathrm{~h}$ of incubation under isothermal condition of $63^{\circ} \mathrm{C}$, and visual evaluation of the outcome could be done. Further-more, LAMP products can be detected by direct fluorescence by adding Loopamp fluorescent detection reagent (FD) (Eiken Chemical Co. Ltd., Japan) at the start of the reaction (Tomita et al., 2008). Other fluorescent dyes such as ethidium bromide, SYBR green and Calcein (Boehme et al., 2007; Abd-Elsalam et al., 2011; Niessen and Vogel, 2010). Evagreen have also been used for visualization of LAMP products under UV light (Poon et al., 2006; Qiao et al., 2007).

Tomlinson et al. (2007) reported on using LAMP for the detection of $P$. ramorum. LAMP assay for the detection of Fusarium spp. is capable of producing the gushing inducing hydrophobin Hyd5p set up (Denschlag et al., 2012). LAMP was used to amplify a 167 bp portion of the acl1 gene in F. tricinctum (Niessen et al., 2012). LAMP primers were able to amplify AMF DNA from colonized carrot roots (Glomus intraradices), and genomic DNA from several taxa of AMF (Gadkar and Rillig, 2008). Dai et al. (2012) development of a LAMP assay targeting the A3aPro element for visual detection of $P$. sojae. LAMP 
amplified 10 times much lower diluted DNA samples of $A$. flavus and $A$. parasiticus than PCR amplification.

A robustness of the specific PCR assay for F. graminearum was high according to the positive PCR amplification obtained with a wide range of template concentration (10 pg-10 ng) using either DNA from pure cultures or from contaminated sample. This method can be used to prepare DNA from numerous wheat grains for molecular analysis with LAMP-PCR method for detecting toxigenic Fusarium collected from cereals.

\section{Conclusion}

The LAMP assay is inexpensive, easy to perform and shows rapid reaction, wherein the amplification can be obtained in $60 \mathrm{~min}$ under isothermal conditions of $63^{\circ} \mathrm{C}$ by employing a set of four species-specific primer mixtures and results can be checked through naked-eye visualization. LAMP reaction can be run using simple lab equipment such as a heating block, water bath, hybridization oven or household equipment, example a microwave oven. In future, we can use these assays for quality control of wheat and other cereals at selected points of entry into Saudi Arabia Gulf cooperation Council states (ports, land borders).

\section{ACKNOWLEDGMENT}

This project was supported by King Saud University, Deanship of scientific research, College of Science Research Centre. We gratefully thank Hans-Peter Klink DSMZ, Germany for providing Fusarium reference strains.

\section{REFERENCES}

Abd-Elsalam KA, Bahkali AH, Moslem MA, Amin O, Niessen L (2011). An optimized protocol for DNA extraction from wheat seeds and loopmediated isothermal amplification (LAMP) to detect Fusarium graminearum contamination of wheat grain. Int. J. Mol. Sci.12: 34593472.

Ahmad MM, Abdin MZ, Javed S (2010). DNA based detection of Aspergillus using LAMP-a green technology for nucleic acid amplification. Int. J. Curr. Chem. 1: 18-22.

Almoammar H (2012). Distribution, characterization and toxigenicity of fungal species isolated from feeds. Ms.C. Thesis, King Saud Univ., Riyadh. p. 156.

Boehme CC, Nabeta PG, Henastroza R, Rubhana Z, Rahim M, Gerhardt E. Sanga M, Hoelscher T, Notomi T, Hase MD, Perkins, R (2007). Operational feasibility of using loop-mediated isothermal amplification (LAMP) for the diagnosis of pulmonary TB in microscopy centres of developing countries. J. Clin. Microbiol. 45: 1936-1940.

Bokhari FM (2010). Implications of fungal infections and mycotoxins in camel diseases in Saudi Arabia. Saudi J. Biol. Sci. 17: 73-81.

Dai TT, Lu CC, Lu J, Dong S, Ye W, Wang Y, Zheng X 2012. Development of a loop-mediated isothermal amplification assay for detection of Phytophthora sojae. FEMS Microbiol. Lett. 334: 27-34.
Denschlag C, Vogel RF and Niessen L (2012). Hyd5 gene-based detection of the major gushing-inducing Fusarium spp. in a loopmediated isothermal amplification (LAMP) assay. Int. J. Food Microbiol. 156: 189-196.

Doohan FM, Brennan J, Cooke BM (2003). Influence of climatic factors on Fusarium species pathogenic to cereals, Eur. J. Plant Pathol. 109: 755-768.

Gadkar V, Rillig MC 2008. Evaluation of loop-mediated isothermal amplification (LAMP) to rapidly detect arbuscular mycorrhizal fungi. Soil Biol. Biochem. 40: 540-543.

Hilton AJ, Jenkinson P, Hollins TW Parry DW (1999). Relationship between cultivar height and severity of Fusarium ear blight in wheat. Plant Pathol. 48: 202-208.

Leslie JF, Summerell BA (2006). The Fusarium Laboratory Manual. Blackwell Publishing Professional, Ames, IA, USA.

Luo J, Vogel RF, Niessen L (2012). Development and application of a loop-mediated isothermal amplification assay for rapid identification of aflatoxigenic molds and their detection in food samples. Int. J. Food Microbiol.159: 214-224.

Niessen L, Vogel R 2010. Detection of Fusarium graminearum DNA using a loop-mediated isothermal amplification (LAMP) assay. Int. J. Food Microbiol.140: 183-191.

Niessen L, Gräfenhan T, Vogel RF (2012). ATP citrate lyase 1 (ac/1) gene-based loop-mediated amplification assay for the detection of the Fusarium tricinctum species complex in pure cultures and in cereal samples. Int. J. Food Microbiol.158: 171-185.

Parida M, Sannarangaiah S, Dash PK, Rao PV, Morita K (2008). Loop mediated isothermal amplification (LAMP): a new generation of innovative gene amplification technique; perspectives in clinical diagnosis of infectious diseases. Rev. Med. Virol. 18: 407-421.

Poon LL, Wong BW, Ma EH, Chan KH, Chow LM, Abeyewickreme W, Tangpukdee N, Yuen KY, Guan Y, Looareesuwan S, Peiris JS (2006). Sensitive and inexpensive molecular test for falciparum malaria: detecting Plasmodium falciparum DNA directly from heattreated blood by loop-mediated isothermal amplification. Clin. Chem. 52: 303-306.

Qiao YM, Guo YC, Zhang XE, Zhou YF, Zhang ZP Wei HP, Yang RF, Wang DB (2007). Loop-mediated isothermal amplification for rapid detection of Bacillus anthracis spores. Biotech. Lett. 29: 1939-1946.

Sun J, Najafzadeh MJ, Vicente V, Xi L, de Hoog GS (2010). Rapid detection of pathogenic fungi using loop-mediated isothermal amplification, exemplified by Fonsecaea agents of chromoblastomycosis. J. Microbiol. Methods 80: 19-24.

Tomita N, Mori Y, Kanda H, Notomi T (2008). Loop-mediated isothermal amplification (LAMP) of gene sequences and simple visual detection of products. Nat. Protoc. 3: 877-882.

Tomlinson JA, Barker I, Boonham N 2007. Faster, simpler, morespecific methods for improved molecular detection of Phytophthora ramorum in the field. Appl. Environ. Microbiol. 73: 4040-4047.

VICAM. (1998). FumoniTest ${ }^{\mathrm{TM}}$ Instruction Manual VICAM, Watertown. p 39.

Xu XM (2003). Effects of environmental conditions on the development of Fusarium ear blight. Eur. J. Plant Pathol. 109: 683-689.

Yli Mattila T, Paavanen-Huhtala S, Parikka P, Konstantinova $\mathrm{P}$, Gagkaeva TY (2004). Molecular and morphological diversity of Fusarium species in Finland and Northwestern Russia, Eur. J. Plant Pathol. 110: 573-584. 